\title{
Capsule Commentary on Paradies et al., 'A Systematic Review of the Extent and Measurement of Healthcare Provider Racism'
}

\author{
Lenny López, MD, MPH \\ Mongan Institute for Health Policy, Massachusetts General Hospital, Brigham and Women's Hospital, Boston, MA, USA.
}

J Gen Intern Med 29(2):362

DOI: $10.1007 / \mathrm{s} 11606-013-2621-\mathrm{Z}$

(c) Society of General Internal Medicine 2013

$\mathrm{R}$ acial and ethnic disparities in health care are well documented, although the reasons underlying them are complex and multidimensional. ${ }^{1}$ The 2002 IOM report, Unequal Treatment, has become the de facto recent reference point for compiling the reasons, mechanisms, and possible approaches to solving this vexing healthcare problem. It defines disparities as differences in the treatment that are not directly attributable to access-related factors, clinical needs, patient preferences, or appropriateness of intervention. Bias leading to discrimination is one of the root causes for disparities that is very difficult to confront as healthcare providers. Not all bias is intentional, conscious or directed at the individual. ${ }^{2}$ Discrimination research has emphasized that bias has a broader context that is not limited to interpersonal factors or encounters but also includes institutional, historic, and socially determined components with complex interplay in how individuals experience, internalize and react to bias. ${ }^{3,4}$

As healthcare providers we want to believe that bias is not something we do and has led to the 'not-me-phenomenon,' referring to clinician beliefs that disparities do exist but not in their own clinical practices. Unfortunately, prior research has demonstrated that physicians do have unconscious beliefs and behaviors that influence the clinical encounter through communication, evaluation and treatment decisions with lasting effects on patient morbidity and mortality. ${ }^{1-3}$

In this issue of JGIM, Pardies et al. present an important contribution to the discrimination research literature by focusing on provider bias. ${ }^{4}$ The article provides a thorough review between 1995 and 2012 including international studies, thus updating and expanding prior reviews. This article highlights several key areas for future research: the need to use multidimensional bias measurement scales; study of other bias-mediating emotions and attitudes (i.e., trust); undertaking longitudinal studies; studying all categories of healthcare providers, not just physicians; and developing interventions that address bias.

There is no reason to lose hope. Prior research has demonstrated effective methods for increasing awareness and self-regulation of implicit biases. ${ }^{1,2}$ Cultural competence and patient-centered interviewing training have also been shown to be effective in addressing bias. ${ }^{1,2,5}$ In summary, the elimination of healthcare disparities is a high priority and addressing all its sources, including bias, is essential to fulfilling our professional goal of providing the highest quality of healthcare for all.

Conflicts of Interest: The author declares that he/she does not have a conflict of interest.

Corresponding Author: Lenny López, MD, MPH; Mongan Institute for Health PolicyMassachusetts General Hospital, Brigham and Women's Hospital, Boston, MA 02114, USA (e-mail: Llopez1@partners.org).

\section{REFERENCES}

1. Smedley BD, Stith AY, Nelson AR, eds. Unequal Treatment: Confronting Racial and Ethnic Disparities in Health Care. Washington: Institute of Medicine, National Academies Press; 2002.

2. Shavers VL, Fagan P, Jones D, Klein WM, Boyington J, Moten C, Rorie E. The state of research on racial/ethnic discrimination in the receipt of health care. Am J Public Health. 2012;102(5):953-966. doi:10.2105/ AJPH.2012.300773.

3. Williams DR, Mohammed SA. Discrimination and racial disparities in health: evidence and needed research. J Behav Med. 2009;32(1):20-47. doi:10.1007/s10865-008-9185-0.

4. Paradies Y, Truong M, Priest N. A systematic review of the extent and measurement of healthcare provider racism. J Gen Intern Med. (Spi \#2583).

5. Sequist TD, Fitzmaurice GM, Marshall R, Shaykevich S, Marston A, Safran DG, Ayanian JZ. Cultural competency training and performance reports to improve diabetes care for black patients: a cluster randomized, controlled trial. Ann Intern Med. 2010;152(1):40-46. doi:10.7326/00034819-152-1-201001050-00009. 